\title{
Nitric oxide repression of Nanog promotes mouse embryonic stem cell differentiation
}

\author{
S Mora-Castilla ${ }^{1}$, JR Tejedo ${ }^{1}$, A Hmadcha², GM Cahuana', F Martín ${ }^{1}$, B Soria ${ }^{2}$ and FJ Bedoya ${ }^{*, 1}$
}

Exposure of mouse embryonic stem (mES) cells to high concentrations of chemical nitric oxide (NO) donors promotes differentiation, but the mechanisms involved in this process at the gene expression level are poorly defined. In this study we report that culture of $\mathrm{mES}$ cells in the presence of $0.25-1.0 \mathrm{mM}$ diethylenetriamine nitric oxide adduct (DETA-NO) leads to downregulation of Nanog and Oct4, the two master genes involved in the control of the pluripotent state. This action of NO was also apparent in the human ES cell line, HS 181. The suppressive action of NO on Nanog gene depends on the activation of p53 repressor protein by covalent modifications, such as pSer15, pSer315, pSer392 and acetyl Lys 379. NO-induced repression of Nanog is also associated with binding of trimethylated histone $\mathrm{H} 3$ and pSer315 p53 to its promoter region. In addition, exposure to $0.5 \mathrm{mM}$ DETA-NO induces early differentiation events of cells with acquisition of epithelial morphology and expression of markers of definitive endoderm, such as FoxA2, Gata4, Hfn1- $\beta$ and Sox 17. This phenotype was increased when cells were treated with valproic acid (VPA) for 10 days.

Cell Death and Differentiation (2010) 17, 1025-1033; doi:10.1038/cdd.2009.204; published online 15 January 2010

Embryonic stem (ES) cells show two principal features: selfrenewal and pluripotency. Extrinsic growth factors, such as leukemia inhibitory factor (LIF), are able to maintain cells in the pluripotent state through the control of master genes at the epigenetic and transcriptional levels. ${ }^{1-4}$ The expressions of Nanog, Oct4 and Sox2 have an important role in the maintenance of the pluripotent state. ${ }^{5}$ Nanog has a critical role in the regulation of cell fate of pluripotent inner cell mass (ICM) during embryonic development, maintaining the pluripotent epiblast and preventing differentiation to primitive endoderm. ${ }^{3}$ High levels of Nanog maintain self-renewal and pluripotency in the absence of LIF. ${ }^{3}$ In addition, Nanog ${ }^{-1-}$ ES cells show slow differentiation to extra embryonic endoderm in vitro, ${ }^{2}$ and Nanog repression leads ES cells to selectively differentiate into primitive endoderm. ${ }^{6}$ On the other hand, Nanog expression is repressed by p53 protein. ${ }^{7}$ During ES cell differentiation, the rapid downregulation of Nanog correlates with the induction of the transcription activity of p53 and p53 ${ }^{\text {Ser15 }}, \mathrm{p} 53^{\text {Ser315 }}$ and p53 ${ }^{\text {Ser392 }}$ phosphorylation. ${ }^{7}$ Modification of $p 53$ activity by knock-in of p53 ${ }^{\text {S315A }}$ results in inefficient Nanog suppression during ESC differentiation. In addition, under oxidative stress conditions, p53 acetylation in residues of lysine K371 and K379 are important to promote translocation of p53 into nucleus. 8,9

Epigenetic modifications of chromatin also have a role in the control of ES cell differentiation. The abundance of transcriptionally active chromatin marks, such as histone H3 trimethylation on lysine $4(\mathrm{H} 3 \mathrm{~K} 4 \mathrm{me} 3)$ and acetylation of histone $\mathrm{H} 4$
(H4Ac), or repressive chromatin marks such as histone $\mathrm{H} 3$ trimethylation on lysine 9 (H3K9me3) in ES cells, supports this notion. ${ }^{10}$ Recently, it has been shown that downregulation of histone demethylase Jmjd2c increases histone H3K9me3 associated with Nanog promoter. This action may facilitate the access of repressors to Nanog promoter during differentiation. ${ }^{11}$

Nitric oxide (NO) has a relevant role during egg activation at fertilization ${ }^{12}$ and it is also involved in developmental processes in the embryo. ${ }^{13,14}$ In vitro differentiation of ES cells to cardiomyocytes upon exposure to high concentrations of chemical NO donors has been reported. ${ }^{15-20}$ The activation of p53 mediates a number of actions of NO in cells, ${ }^{21-23}$ but the involvement of p53 in the activation of differentiation in ES cells by NO remains to be determined. . $^{84}$

In this context, we describe in this study for the first time that short treatment with high concentrations of the chemical $\mathrm{NO}$ donor, diethylenetriamine nitric oxide adduct (DETA-NO), leads to Nanog and Oct4 mRNA decrease and protein downregulation, upregulation of p53 expression and promotion of p53 post-translational modifications. NO treatment also modifies the patterns of methylation and acetylation of $\mathrm{H} 3$ and $\mathrm{H} 4$ histones. Moreover, we show that p53 phosphorylated on Ser 315 binds to Nanog promoter in the absence of LIF, favoring its repression. Exposure to NO donor for $19 \mathrm{~h}$ also induces differentiation events that are apparent by the presence of cells with epithelial morphology, and by the expression of early endoderm markers such as Pdx-1. When these cells were exposed to the differentiation protocols for 10

\footnotetext{
${ }^{1}$ Andalusian Center for Molecular Biology and Regenerative Medicine (CABIMER)-University Pablo de Olavide, CIBERDEM, Seville, Spain and ${ }^{2}$ Andalusian Center for Molecular Biology and Regenerative Medicine (CABIMER)-Fundación Progreso y Salud, Seville, Spain

${ }^{*}$ Corresponding author: FJ Bedoya. Av. Américo Vespucio s/n-Edif, CABIMER, Parque Científico y Tecnológico Cartuja 93, 41092 Seville, Spain. Tel: + 34954467840 ; Fax: + 34954 461664; E-mail: francisco.bedoya @ cabimer.es

Keywords: embryonic stem cell; self-renewal; differentiation; Nanog; oct4 repression; nitric oxide

Abbreviations: NO, nitric oxide; LIF, leukemia inhibitory factor; $\mathrm{H} 3 \mathrm{~K} 4 \mathrm{me} 3$, histone $\mathrm{H} 3$ trimethylation on lysine 4; $\mathrm{H} 3 \mathrm{~K} 9 m e 3$, histone $\mathrm{H} 3$ trimethylation on lysine 9; $\mathrm{H} 4 \mathrm{Ac}$, acetylation of histone H4; DETA-NO, diethylenetriamine nitric oxide adduct; VPA, valproic acid

Received 06.4.09; revised 10.11.09; accepted 19.11.09; Edited by R De Maria; published online 15.1.10
} 
days, cells with endoderm phenotype and expressing definitive endoderm markers, such as FoxA2, Sox17, Hnf1- $\beta$ and GATA4, were obtained.

\section{Results}

Nitric oxide induces Nanog and Oct4 downregulation. When mES cells (line D3) were exposed to DETA-NO, a dose-dependent downregulation of Nanog and Oct4 protein expression was apparent (Figure 1a). In addition, the decrease in Nanog and Oct4 was time dependent on cells exposed to $1 \mathrm{mM}$ DETA-NO (Figure $1 \mathrm{~b}$ ). This effect is linked to repression of Nanog and Oct4 genes as evidenced by measurement of mRNA levels with quantitative PCR (Figure 1d). The repressive action of NO on these two genes was also apparent in other mESC cells lines, such as R1-E and AINV-15, both at the protein and mRNA level (Figures 1c and e, respectively) and in the hES cell line HS-181 (Figure 1f). Treatment of D3 mES cells expressing GFP driven by Oct4 promoter with $1 \mathrm{mM}$ DETA-NO causes a reduction in GFPpositive cells of 82.15 and $95.75 \%$ in the presence and absence of LIF, respectively, and this effect is accompanied by changes in colony morphology (Supplementary Figure 1).

p53 is upregulated by nitric oxide. Treatment of mES cells with DETA-NO induces a dose-dependent increase in p53 protein, and this action is more relevant in the absence of LIF (Figure 2a). The level of p53 transcript is not altered by DETANO (Figure 2b). In contrast, a prominent transactivation of p21 is observed, either in the presence or absence of LIF. The transcription of additional Nanog repressors, such as Tcf3 and mSin3a, was not affected by DETA-NO treatment in the presence of LIF (Figure 2b). In the absence of LIF, Tcf3 transcription was increased and DETA-NO treatment decreased Tcf3 and did not modify mSin3A level of transcription (Figure 2b).

Nitric oxide induces p53 and H3 histone post-translational modifications. Figure $3 a$ shows that exposure of D3 cells to DETA-NO increases p53 protein expression and promotes p53 phosphorylation in Serine 15 and Serine 392, both in the presence and absence of LIF, and acetylation in Lys 379 in the absence of LIF in a concentration-dependent manner. Trimethylation of $\mathrm{H} 3$ histone in lysine 9 and mono/di and tri-methylation in lysine 4 is increased after DETA-NO treatment. On the other hand, DETA-NO produces a slight increase in monomethylation of histone 3 in lysine 4 , and acetylation of histones $\mathrm{H} 3$ and $\mathrm{H} 4$ (Figure $3 \mathrm{~b}$ ). These actions are again more apparent in the absence of LIF. Moreover, a partial stabilization of p53 is obtained when cells are cultured in the presence of the proteasome inhibitor lactacystin (Supplementary Figure 4). Under this condition, phoshorylation of S15, S315 and S392 is negligible in untreated cells. Exposure to DETA-NO leads to significant increases in S315 and S392 phosphorylations.

Treatment with nitric oxide enhances p53 nuclear protein level and repression of Nanog promoter. Treatment with DETA-NO induces an increase of p53 protein in nucleus, both a

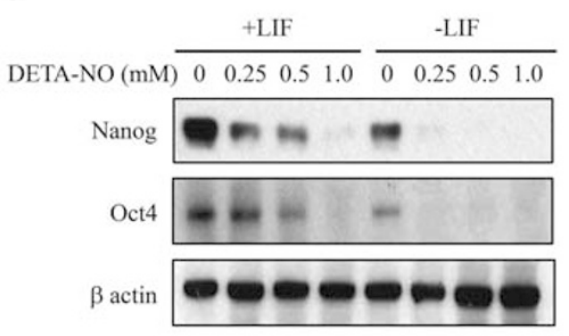

d

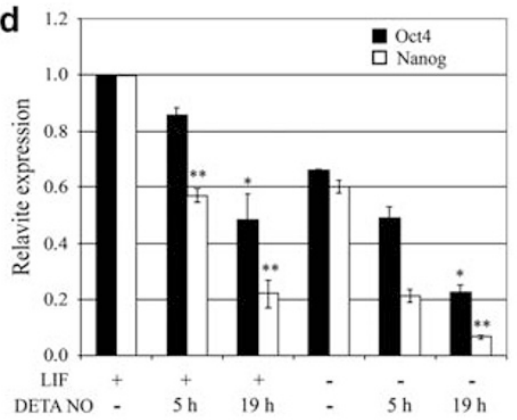

b
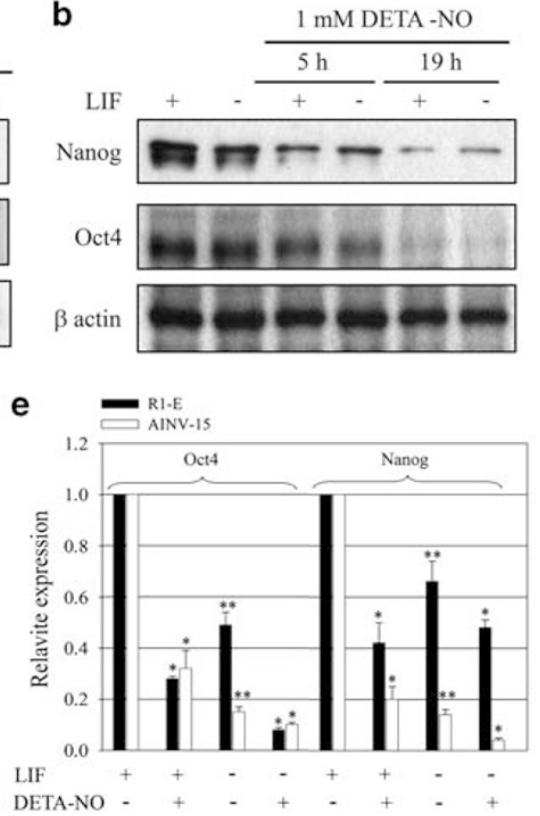

C

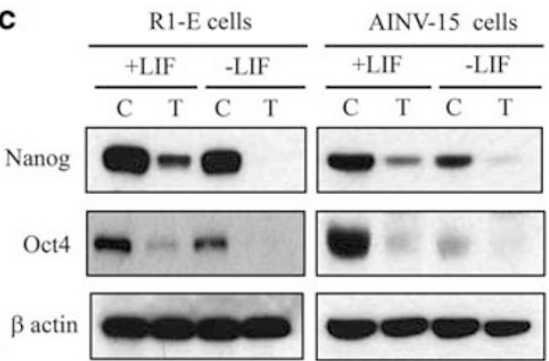

f

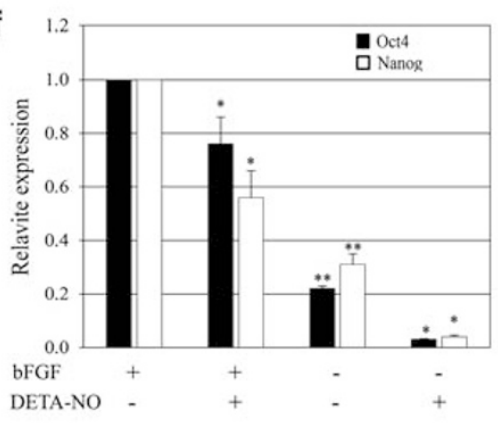

Figure 1 Nanog and Oct4 downregulation by DETA-NO. (a) Dose dependency of DETA-NO. mES cells (D3 line) were exposed for $19 \mathrm{~h}$ to $0.25,0.5$ and $1 \mathrm{mM}$ of the NO donor in the presence or in the absence of LIF. (b) Time dependence of DETA-NO. D3 cells were exposed for 5 and $19 \mathrm{~h}$ to $1 \mathrm{mM}$ DETA-NO in the presence or absence of LIF. (c) Effect of DETA-NO exposure on R1-E and in AINV-15 mES cells. C, control, non-exposed cells; T, cells exposed to $0.5 \mathrm{mM}$ DETA-NO for $19 \mathrm{~h}$. (d) Real-time PCR analysis of Nanog and Oct4 expression in D3 cells. (e) qPCR analysis of R1-E and in AINV-15 mES cells. (f) qPCR analysis of HS181 human ES cell line. In (a-c) Nanog and Oct4 were detected using western blot according to the protocol described in Materials and Methods. Blots are representative of three independent experiments. In ( $d-f$ ) analyses using real-time PCR were made according to the protocol described in Materials and Methods. Data are mean \pm S.D. of three independent experiments. (d, e $)^{*}$ Significant difference from respective control without treatment for Oct4 expression, $P<0.050$; * significant difference from respective control without treatment for Nanog expression, $P<0.001$. (f) *Significant difference from respective control without treatment $P<0.001$; ${ }^{* *}$ significant difference from control with bFGF $P<0.001$ 

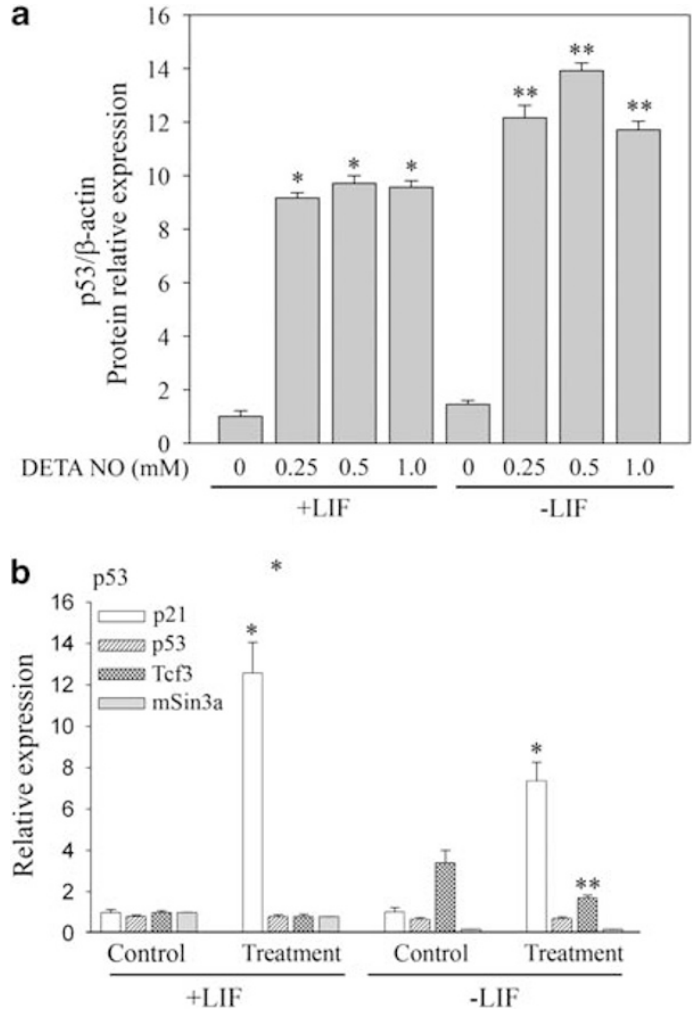

Figure 2 p53 upregulation and $p 53$ activation induced by nitric oxide. $\mathrm{mES}$ cells were exposed for $19 \mathrm{~h}$ to $0.25,0.5$ and $1 \mathrm{mM}$ of DETA-NO in the presence or absence of LIF. (a) p53 upregulation by DETA-NO. Densitometric analysis of p53 and $\beta$-actin protein blots. Data are mean \pm S.D. of 3-5 independent experiments. *Significant difference from control, untreated cells in the presence of LIF, $P<0.001$; ** significant difference from control, untreated cells in the absence of LIF, $P<0.001$. (b) Real-time PCR analysis of p21, MDM2, p53, Tcf3 and mSin3a expression. Analysis by real-time PCR was made according to the protoco described in Materials and Methods. Data are mean \pm S.D. of three independent experiments ("significant difference with respect to control with LIF, $P<0.001$; ${ }^{*}$ significant difference with respect to control without LIF conditions; $P<0.001$ )

in the presence and absence of LIF (Figure 4a), which is associated with Nanog downregulation. Nanog is located mainly in the nucleus and exposure to the NO donors leads to a substantial decrease in the amount of this protein in nucleus. Figure $4 \mathrm{~b}$ shows that p53 phosphorylated in Ser 392, Ser 315 and Ser 15 after exposure of D3 cells to DETA-NO is located only in nucleus. In contrast, exposure of D3 cells to the apoptosis inducer camptothecin leads to p53 gene expression, Ser 15 phosphorylation and translocation of the protein to mitochondria (Figure 4c).

Using ChIP assay, we document that $\mathrm{H} 3$ histone trimethylated in Lys 9 binds to proximal and distal Nanog promoter after DETA-NO treatment, and these interactions were observed both in the absence and presence of LIF (Figure 4d). In addition, binding of p53 and p53 phosphorylated in Ser 315 to p53 element of Nanog promoter was observed only when cells were cultured in the absence of LIF (Figure 4e). p53 binding to Nanog promoter was confirmed with two different antibodies (Santa Cruz Biotechnology, Santa Cruz, CA, USA, FL-393; and Calbiochem, San Diego, $\mathrm{CA}, \mathrm{USA}, \mathrm{Ab}-7)$ and nonspecific binding of p53 antibodies to a

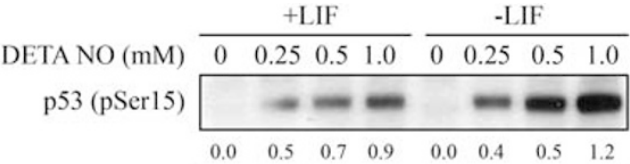

p53 (pSer392

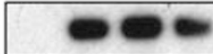

$\begin{array}{llllllll}0.0 & 1.5 & 1.8 & 1.2 & 0.0 & 0.2 & 0.3 & 1.6\end{array}$

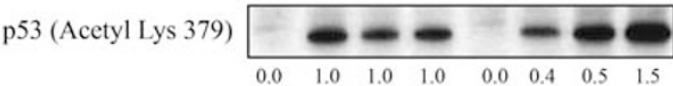

p53

$\begin{array}{llllllll}0.0 & 1.0 & 1.0 & 1.0 & 0.0 & 0.4 & 0.5 & 1.5\end{array}$

b

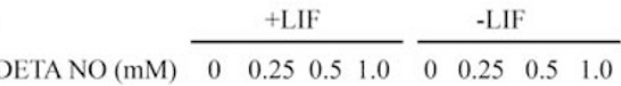

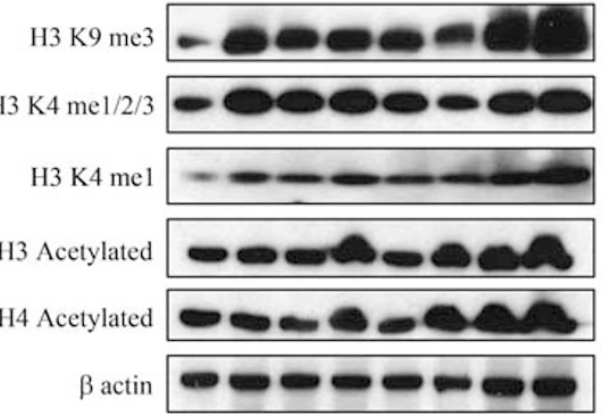

Figure 3 Nitric oxide increases p53 and histone post-translational modifications. D3 mES cells were exposed for $19 \mathrm{~h}$ to $0.25,0.5$ and $1 \mathrm{mM}$ DETA-NO in the presence or absence of LIF. (a) p53 post-translational modifications induced by DETA-NO, p53 (phospho Ser 15), p53 (phospho Ser 392) and p53 (acetyl Lys 379) and $\beta$-actin were detected using western blot according to the protocol described in Materials and Methods. Numbers below blots denote the differences between densitometry values of respective phosphorylation in p53 and p53 protein levels. (b) $\mathrm{H} 3$ and $\mathrm{H} 4$ histone post-translational modifications induced by DETA-NO. Histone $\mathrm{H} 3$ trimethylated in lysine 9 (H3K9me3), histone $\mathrm{H} 3$ mono/di/trimethylated in lysine 4 ( $\mathrm{H} 3 \mathrm{~K} 4 \mathrm{me} 1 / 2 / 3$ ) histone $\mathrm{H} 3$ acetylated, histone $\mathrm{H} 4$ acetylated and $\beta$-actin were detected using western blot according to the protocol described in Materials and Methods. Blots are representative of at least three independent experiments

p53-nonspecific sequences of Nanog exon 4 region of chromatin was not observed (data not shown).

Depletion of p53 cancels the effects of nitric oxide on Nanog repression, apoptosis and differentiation. Transfection of D3 mES cells with p53 shRNA leads to depletion of p53 protein signal (Figure $5 \mathrm{a}$, upper) and partially cancels the repressive action of DETA-NO on Nanog expression (Figure 5a, middle). Exposure to $1 \mathrm{mM}$ DETA-NO leads to induction of apoptosis in D3 mES cells, as detected by the loss of intact PARP and by caspase 3 cleavage (Figure $5 \mathrm{~b}$ ). This action is not apparent in p53-interfered cells. On the other hand, DETA-NO treatment increases the expression of the differentiation markers, GATA6 and FoxA2 (Figure 5c) and the transactivation of p21 (Figures $2 b$ and $5 \mathrm{c}$ ). Depletion of p53 significantly reduces the effect of NO on the expression of Gata 6, FoxA2 and p21 genes (Figure $5 \mathrm{c}$ ).

Nitric oxide treatment results in cell populations resistant to apoptosis and with endoderm phenotype. Treatment with $0.5 \mathrm{mM}$ DETA-NO for $19 \mathrm{~h}$ induces apoptosis in $40 \%$ of adherent cells, as determined by incorporation of Annexin $\mathrm{V}$ 


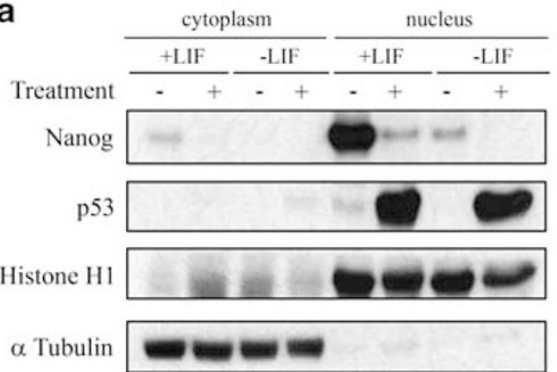

d

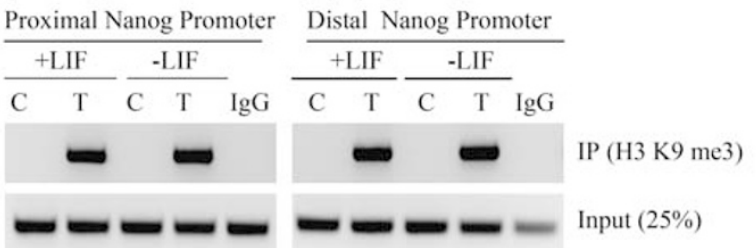

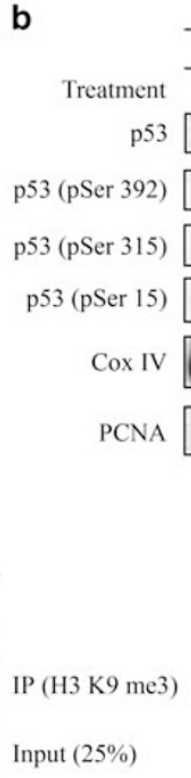

mitochondria $\frac{+ \text { LIF }}{-+} \frac{\text { LIF }}{-++}$
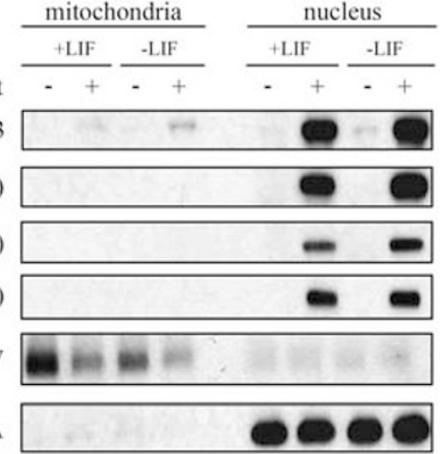

e

e

$\frac{\begin{array}{c}\text { p53 element } \\ \text { (Nanog promoter) }\end{array}}{\frac{\text { +LIF }}{\mathrm{C} \quad \mathrm{T}} \frac{\text {-LIF }}{\mathrm{C} \text { T }}}$

IP (p53)

Input (25\%)
C

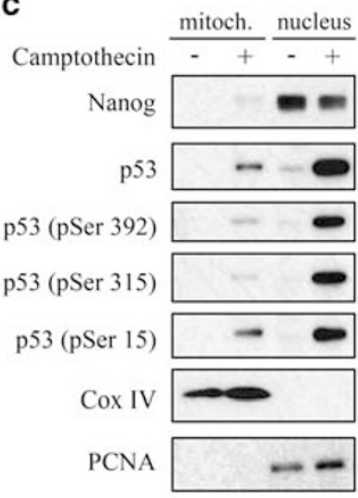

p53 element (Nanog promoter)

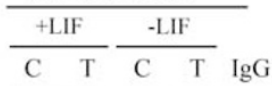

Figure 4 Nitric oxide promotes p53 translocation from cytoplasm to nucleus and recruitment of trimethyl histone $\mathrm{H} 3$ to proximal and distal Nanog promoters and p53 in p53 element of Nanog promoter. D3 mES cells were exposed for $19 \mathrm{~h}$ to $1 \mathrm{mM}$ DETA-NO (Treatment) in the presence or absence of LIF. (a) Western blot analysis of p53 translocation. Nanog, p53, histone $\mathrm{H} 1$ and $\alpha$-tubulin were detected in cytoplasmatic and nuclear fractions using western blot according to the protocol described in Material and Methods. Histone $\mathrm{H} 1$ is used as control for nuclear fraction. $\alpha$-Tubulin is used as control for cytoplasmatic fraction. (b) Effect of DETA-NO treatment on subcellular localization of p53. Cells were exposed to $1 \mathrm{mM}$ DETA-NO for $19 \mathrm{~h}$ and the phosphorylation status of p53 in mitochondria and in nuclear fraction were detected using western blot. Cox IV is used as control for mitochondria fraction and PCNA as control for nuclear fraction. (c) Effect of exposure of D3 cells to the apoptosis inducer camptothecin on p53 phosphorylation and subcellular localization. D3 cells were cultured in the presence of LIF and exposed for $19 \mathrm{~h}$ to $1 \mu \mathrm{M}$ camptothecin. Proteins were detected using western blot. (d) Chip analysis shows binding of histone $\mathrm{H} 3$ trimethylated in Lys 9 to the proximal and distal Nanog promoter. IgG mock control was performed under NO treatment. (e) Chip analysis shows binding of p53 and p53 (phospho Ser 315) to p53 element of Nanog promoter. Treatment: IgG mock control was performed under NO treatment. Chip assays were realized according to the protocols described in Materials and Methods. Blots are representatives of three independent experiments
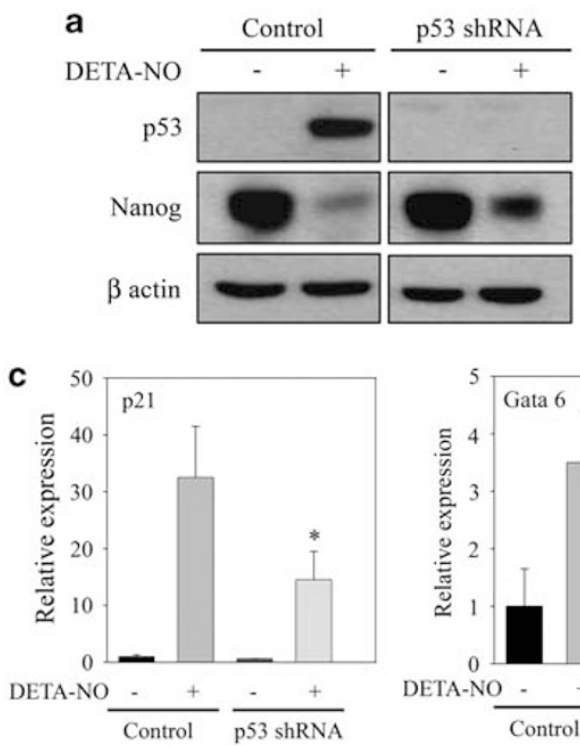
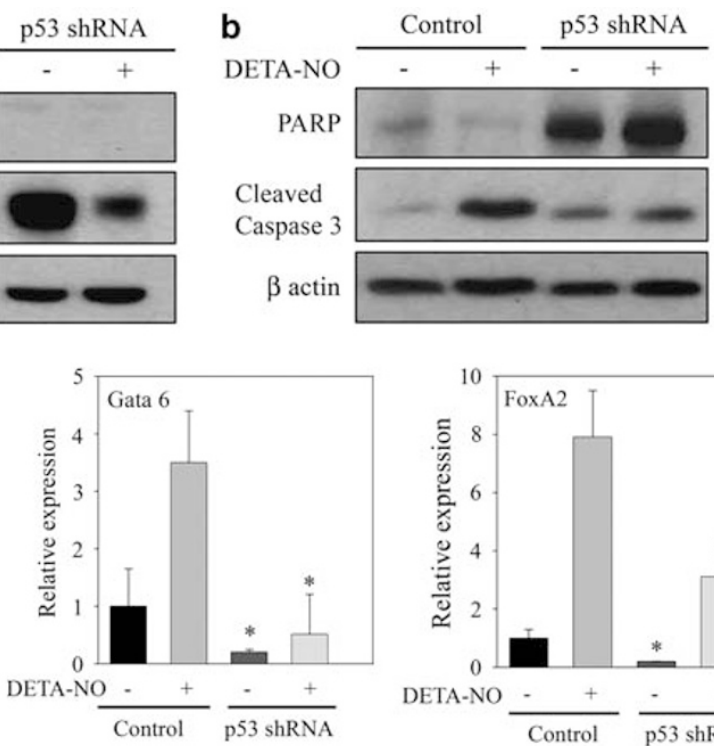

Figure 5 p53 depletion leads to recovery of Nanog expression and prevents apoptosis and differentiation induced by nitric oxide. D3 mESC control and transfected with control and p53 shRNA were cultured in medium with LIF and exposed for $19 \mathrm{~h}$ to $1 \mathrm{mM}$ DETA-NO. (a) Effect on Nanog repression induced by NO. Nanog, p53 and $\beta$ actin were detected using western Blot according to the protocol described in Material and Methods. (b) Effect on apoptosis induced by NO. PARP (band of $116 \mathrm{kDa}$ ), cleaved caspase 3 (band of $18 \mathrm{kDa}$ ) and $\beta$-actin were detected using western Blot according to the protocol described in Materials and Methods. (c) Effect on p53 transactivation activity, and differentiation markers. Real-time quantification of p21, Gata 6 and FoxA2. Analysis using real-time PCR was made according to the protocol described in Materials and Methods. Blots are representative of at least three independent experiments. Data are mean \pm S.D. of three independent experiments. ${ }^{*}$ Significant difference with respect to control with LIF without p53 shRNA condition 
A

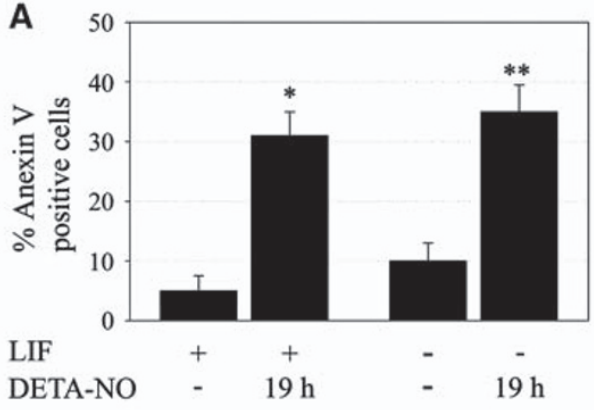

C

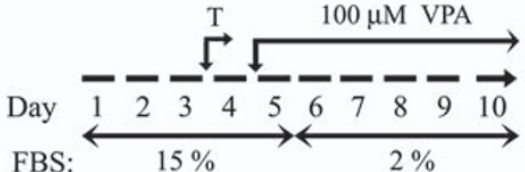

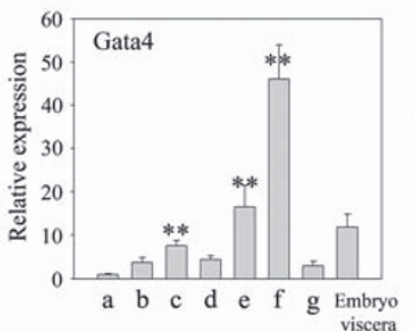
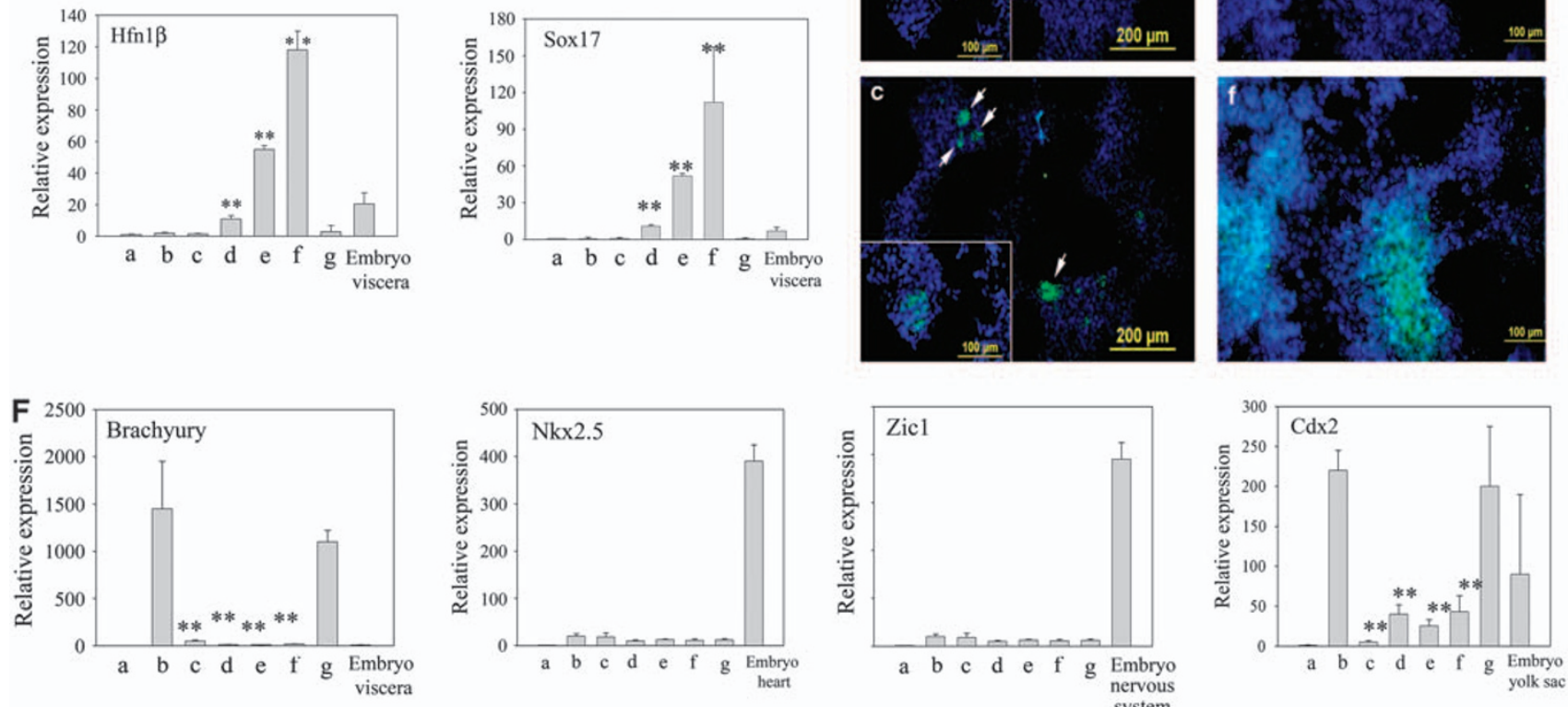

Figure 6 Nitric oxide induces differentiation to endoderm. D3 cells were exposed for $19 \mathrm{~h}$ to $0.5 \mathrm{mM}$ DETA-NO in the presence or absence of LIF. (A) Nitric oxide induction of apoptosis in adherent cells. Apoptosis was measured by binding of Annexin V to cell surface. (B) Real-time quantification of Hsp 70 and Hmox 1 expression induced by nitric oxide (C) Protocol for mES cells differentiation. Cells were cultured for 3 days in the absence of LIF and treated for $19 \mathrm{~h}$ with $0.5 \mathrm{mM}$ DETA-NO. Cells were then cultured in medium containing $15 \%$ fetal bovine serum (FBS) in the absence of LIF and valproic Acid (VPA). From day 6 to day 10, cells were maintained in media containing $2 \%$ of FBS and $100 \mu \mathrm{M}$ VPA. (D) Fluorescent images from cells positive for Pdx-1 and Foxa 2 in cells treated with $0.5 \mathrm{mM}$ DETA-NO for $19 \mathrm{~h}$ (a-c) and from cells collected at day 8. Images (d-f) are from cells counter-stained with DAPI. (E) Real-time quantification of definitive endoderm markers (FoxA2, Gata 4, Hnf1- $\beta$ and Sox 17). (F) Real-time quantification of mesendoderm (brachyury), mesoderm (Nkx2.5), ectoderm (Zic1) and trofectoderm (Cdx2) markers. Positive controls for endoderm, mesoderm, ectoderm and trofectoderm were from 10-day embryo viscera, heart, nervous system and yolk sac, respectively. In $(E)$ and $(F)$ a is control with LIF; $b$ is control, 4 days without LIF; c is 3 days without LIF plus DETA-NO treatment for $19 \mathrm{~h}$; $d$ is c plus 2 days with VPA; $e$ is c plus 4 days with VPA; $f$ is c plus 6 days with VPA and $g$ is control, 10 days without LIF. Data are mean \pm S.D. of three independent experiments. (A, B, E and F) ${ }^{\star}$ Significant difference with respect to control with LIF, $P<0.001$; ** significant difference with respect to control without LIF, $P<0.001$. (D) Images are representative of three independent experiments 
into cell surface, both in the absence and presence of LIF (Figure 6A). Of the adherent population of cells, $60 \%$ are Annexin $\mathrm{V}$ negative and manage to escape from DETA-NOinduced cell death. Colonies of apoptosis-resistant cells show different morphologies when compared with colonies of control cells (data not shown) and show areas expressing endoderm markers, such as $\mathrm{Pdx} 1$ (Figure 6D, a-c). Moreover, these adherent cells have enhanced expression of genes encoding for cytoprotective proteins, such as hemeoxigenase-1 (Hmox1) and heat shock protein 70 (Hsp70) (Figure 6B).

Adherent cells after DETA-NO treatment were subsequently exposed to a newly designed protocol for endoderm differentiation (Figure 6C). This protocol consists of four steps: (i) precondition step, in which cells grow in the absence of LIF for 3 days; (ii) selection step, in which cells are exposed to $0.5 \mathrm{mM}$ DETA-NO for $19 \mathrm{~h}$; (iii) prematuration step, in which cells are cultured for $24 \mathrm{~h}$ in media containing $15 \% \mathrm{FBS}$ and $100 \mu \mathrm{M}$ VPA and (iv) maturation step, in which cells are exposed from day 6 to day 10 to media containing $2 \%$ FBS and $100 \mu \mathrm{M}$ VPA. Maturation led to generation of a population of cells with definitive endoderm fate. Figure 6D shows different stages in the morphology of cells during the differentiation process. DETA-NO-treated D3 mES cells show endoderm morphology and express endoderm markers such as Gata 4 (Figure 6E, condition c) and Pdx1 (Figure 6D, a-c); similar results are observed in R1-E and AINV-15 mES cells (Supplementary Figure 3). Other endoderm markers, such as Fox A2, Hnf1B and Sox 17, do not change significantly (Figure $6 \mathrm{E}$, condition $\mathrm{c}$ ). Figure $6 \mathrm{E}$, conditions $\mathrm{d}-\mathrm{f}$, also shows expression of definitive endoderm markers obtained in steps iii and iv of the protocol described earlier. At the end of treatment, FoxA2, Gata4, Hnf1- $\beta$ and Sox 17 increase 7.815.3- 42.1- and 120-fold, respectively, when compared with control without treatment. After 8 days of treatment, all cells express FoxA2 marker (Figure 6D, d-f). Markers of mesendoderm (Brachyury), mesoderm (Nkx2.5), ectoderm (Zic 1) and trofectoderm $(\mathrm{Cdx} 2)$ were not detected at the end of treatment (Figure 6F).

\section{Discussion}

We show in this report that high levels of NO rapidly induces repression of self-renewal genes and starts the differentiation process in the D3 line and in other mESC cells lines. In this context, previous reports have shown that exposure to high levels of chemical NO donors halts proliferation events and promotes differentiation of embryonic cells into cardiomyocytes, ${ }^{15-20}$ but no evidence was provided on the molecular mechanisms underlying the regulation of pluripotency by $\mathrm{NO}$ at the gene transcription level.

The regulation of Nanog and Oct4 levels is a key switch of ES cell fate. ${ }^{25,26}$ The presence of these transcription factors maintain self-renewal and pluripotency. ${ }^{26}$ During differentiation process Nanog and Oct4 levels decrease significantly. 6,27 In this study we show that treatment with high levels of DETANO induce downregulation of Nanog and Oct4. This effect is apparent after short periods of exposure $(19 \mathrm{~h})$ and is rapid when compared with other systems, such as Nutlin (3 days) ${ }^{28}$ and Activin A plus Wnt ( 3 days), ${ }^{29-31}$ a feature that makes it suitable for use in differentiation protocols.

In mES cells, Nanog and Oct4 protein and mRNA downregulation by $\mathrm{NO}$ is dose and time dependent, both in the presence and absence of LIF (Figures 1a-e). Nanog and Oct4 mRNA was also downregulated by NO in hES cells cultured in the presence or absence of bFGF (Figure 1f). The fact that NO decreases Nanog and Oct4 mRNA significantly (Figures 1d-f) suggest the existence of regulatory actions at the gene expression level (Figures 1d-f). In this context, an increase in p53 suppressor of Nanog was observed (Figures 2a and 3a). On the other hand, NO increases p53 stability and induces p53 activation through post-translational modifications, such as p53 phosphorylation in Ser15, Ser392, acetylation in Lys 379 (Figure 3a) and phosphorylation in Ser 315 (Figure 4b). According to previous reports, these modifications have three consequences: (1) phosphorylation in Ser 15 increases p53 stability and participates in the recruitment of factors that promote C-terminal acetylation. ${ }^{32}$ (2) acetylation of Lys 379 in ES cells promotes p53 translocation into nucleus, ${ }^{8}$ and (3) phosphorylation of Ser315 increases its transcriptional activity. ${ }^{33}$ Our results show that NO treatment enhances p53 protein stability (Figures $2 a$ and $3 a$ ), increases p53 acetylation (Figure $3 a$ ), promotes p53 translocation into nucleus associated with its acetylation (Figures $4 a$ and b), increases transcriptional activity and induces p21 transactivation (Figure $2 b$ ). It is interesting to note that p53 activated by NO through phosphorylation in Ser 315 can directly bind to p53 element present in Nanog promoter (Figure 4e). These results are in agreement with a previous report showing that knock-in of p53 ${ }^{\mathrm{S} 315 \mathrm{~A}}$ results in inefficient Nanog suppression. ${ }^{7}$ In this study we show for the first time that phosphorylated p53 ${ }^{\mathrm{S} 315}$ after exposure of cell to DETA-NO binds to Nanog promoter and represses Nanog gene (Figure 4e). On the other hand, NO repression of Nanog is specifically dependent on p53, as other repressors of Nanog, such as Tcf3 and mSim3a, are not upregulated (Figure 2b). Moreover, p53 depletion with p53 shRNA cancels significantly the effect of NO on Nanog repression (Figure 5a).

It has been reported that p53 phosphorylation in Ser 15 and Ser 392 induced by oxidative stress is involved in p53dependent apoptosis. ${ }^{8}$ Exposure to high levels of the chemical NO donor also triggers apoptotic events in these cells. In fact, $30-40 \%$ of cells are Annexin V positive, which is a sign of apoptosis (Figure 6A). Other apoptosis markers, such as PARP degradation and cleaved caspase-3, are also apparent in these cells (Figure 5a and Supplementary Figure $2)$. These signs of apoptosis are accompanied by a rise in p53 protein levels (Figure 2a and Supplementary Figure 2). Nevertheless, a population of cells expressing citoprotective genes, such as heme-oxigenase-1 and Hsp70 (Figure 6b), manage to resist the oxidative stress and likely enter the differentiation program. In cells that remain adherent after NO treatment, p53 ${ }^{\text {pSer15,pSer315,pSer392 is located in nucleus }}$ (Figure $4 b$ ). On the other hand, treatment of cells with camptothecin, a genotoxic stress agent that promotes p53 translocation to mitochondria and nucleus, ${ }^{34}$ leads to detection of p53 in nucleus with a similar pattern of phosphorylation in Ser residues, but $\mathrm{p} 53^{\mathrm{pSer} 15}$ is also present in mitochondrial fractions, in agreement with previous reports. ${ }^{8,34}$ It is entirely 
possible that oxidative stress triggered by $\mathrm{NO}$ induces a rise in p53 protein levels, perhaps through post-translational changes in the protein such as those reported in this study. Mitochondria have a prominent role in apoptosis triggered by $\mathrm{NO}$ and the presence of p53 in this organelle may contribute to regulate some of the steps involved in this pathway. Nuclear actions of p53 during apoptosis are also well known. The inhibition of p53 by specific shRNA caused inhibition of PARP degradation and caspase 3 cleavage. The fact that exposure of ES cells to apoptotic agents, such as staurosporine, triggers apoptotic events, such as PARP degradation, caspase 3 cleavage and p53 upregulation without affecting Nanog expression, supports the notion that Nanog downregulation induced by high $\mathrm{NO}$ concentrations is independent of the apoptotic actions induced by this molecule.

Interestingly, treatment with $\mathrm{NO}$ causes a significant increase in $\mathrm{H} 3 \mathrm{~K} 9$ me3 (Figure 3b), which is related with Nanog repression. ${ }^{11}$ There is also recruitment of histone H3K9 me3 in Nanog promoter after NO treatment (Figure $4 d$ ). It is particularly relevant that depletion of histone demethylase Jmjd2c induces H3K9 me3 downregulation of Nanog and induction of endodermal markers such as Gata4 and Gata6. ${ }^{11}$ In this respect, we have observed specific binding of histone H3K9 me3 on Nanog promoter, and subsequent expression of endoderm markers, such as FoxA2, Gata4, Hnf1- $\beta$ and Sox17, when cells are stimulated to differentiate with NO (Figure 6e). Moreover, NO increases acetylation of histones $\mathrm{H} 3$ and $\mathrm{H} 4$ (Figure $3 \mathrm{~b}$ ). According to these results, NO changes the structure of chromatin in ES cells, and this phenomenon is related with the starting of differentiation programs ${ }^{10,11}$ and cell memory. ${ }^{35}$ On the other hand, treatment with NO allows us to evaluate the role of p53 in the differentiation process. In this context, NO increases the expression of differentiation genes, such as Gata 6 and FoxA2, and depletion of p53 cancels significantly the effect of $\mathrm{NO}$ (Figure $5 \mathrm{c}$ ). These results emphasize the role of p53 in the differentiation process in ES cells in accordance with previous reports. ${ }^{28}$

According to the results reported in this study and in agreement with published data indicating that Nanog repression is required for endoderm differentiation ${ }^{6}$ and that histone modifications are required for correct differentiation, ${ }^{10,11}$ we have developed a protocol for stem cell differentiation into definitive endoderm. The protocol consists of a 'precondition step' for 3 days in LIF-starved conditions, followed by a 'selection step' by NO treatment for $19 \mathrm{~h}$, a 'pre-maturation step' with VPA in 15\% FBS for $24 \mathrm{~h}$ and 'maturation step' with VPA in $2 \%$ FBS for 5 days. In the second step, NO downregulates Nanog and eliminates cells not committed to differentiate. Selected adhered cells continue to differentiate with VPA, which is a selected factor used for endoderm differentiation and maturation, during pancreatic development. $^{36}$ To confirm this fact, it has been shown that histone deacetylase inhibitors have an important role in mES cell differentiation. ${ }^{36-38}$ It is interesting to note that Brachyury was highly expressed in control cells without LIF condition, indicating that these cells were committed to differentiation to mesoderm and endoderm. The fact that Brachyury expression was cancelled and $\mathrm{Pdx} 1$ and Gata 4 increased by $\mathrm{NO}$ treatment indicates that $\mathrm{NO}$ favored differentiation to endoderm. At the end of this differentiation protocol, a

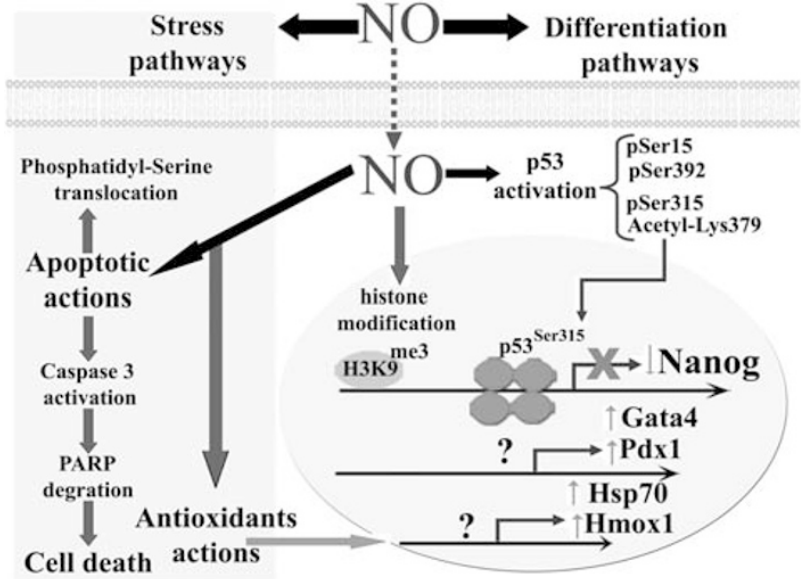

Figure 7 NO actions in mES cells. Exposure of mES cells to high levels of NO activates two pathways: (i) A stress pathway that triggers the death program and also elicits a survival response with enhanced expression of the antioxidant genes Hsp70 and Hmox1 and (ii) a differentiation pathway that promotes differentiation to endoderm. This event is linked to repression of self-renewal genes, such as Nanog, and expression of differentiation genes, such as Gata4 and Pdx1

population of cells differentiated to definitive endoderm and expressed high levels of FoxA2, Gata4, Hnf1- $\beta$ and Sox 17. On the other hand, it has been shown that NO signalling is implicated in cardiomyocyte differentiation. ${ }^{15-20}$ Exposure of ES cells to different NO donors, ${ }^{15,19,20}$ or overexpression of inducible $\mathrm{NOS}^{15}$ in these cells, increases the number of beating cardiomyocytes. In these reports, expression of cardiac markers was already detected in embryoid bodies. Subsequent exposure of these structures to high NO increases the number of beating cardiomyocytes. The default program in embryoid bodies is to differentiate to multiple cellular derivatives, including cardiomyocytes, and NO increases differentiation events by suppressing undifferentiated signals in the cells. In our model, culturing ES cells in adherent conditions and withdrawal of LIF allows us to equally expose cells to NO and analyze the action of this agent on pluripotency markers that are still expressed.

The scheme presented in Figure 7 summarizes the effect of high concentrations of $\mathrm{NO}$ in mES cells, as derived from the results reported in this study. NO triggers two major pathways: (i) stress pathways that select cells resistant to stress through increase in antioxidant genes and promote death by apoptosis of cells that fail to express these genes; (ii) differentiations pathways that promote cell differentiation through repression of self-renewal genes, such as Nanog, and increase in differentiation genes such as Gata4 and Pdx1. Deciphering the complete set of transcription factors targeted by $\mathrm{NO}$ and regulating the expression of differentiation and antioxidant genes deserve further exploration.

In summary, we have designed a novel approach for embryonic stem cell differentiation to definitive endoderm. High levels of NO induce a switch-off of two of the most important genes of pluripotency, Nanog and Oct4, enhance transactivation activity of $\mathrm{p53}$, remodel chromatin and promote expression of early endoderm markers (Pdx1 and Gata 4). The addition of VPA leads to differentiation to definitive endoderm. This protocol might be instrumental for 
the generation of progenitors of endoderm-derived tissues such as liver or pancreas.

\section{Materials and Methods}

Cell culture and transfection. Four embryonic stem cell lines were used: (1) mESC line D3, R1-E and AINV-15, obtained from the ATCC, and (2) hESC line HS181, kindly provided by the Karolinska Institute..$^{39}$

Feeder-free D3 mES cells were cultured on Nunclon surface dishes, R1-E and AINV-15 mES cell were cultured on gelatinized dishes. All cells were maintained at $37^{\circ} \mathrm{C}$ with $5 \% \mathrm{CO}_{2}$ in Dulbecco's modified Eagle's medium (DMEM; Gibco, Carlsbad, CA, USA), supplemented with $15 \%$ heat-inactivated fetal bovine serum (Hyclone, Logan, UT, USA), $0.5 \mathrm{mM} \beta$-mercaptoethanol (Gibco), $2 \mathrm{mM} \mathrm{L-glutamine} \mathrm{(Gibco),}$ $0.1 \mathrm{mM}$ MEM nonessential amino acid (Gibco), $5000 \mathrm{U} / \mathrm{ml}$ penicillin/streptomycin (Gibco) and $1000 \mathrm{U} / \mathrm{ml}$ LIF (ESGRO, Chemicon, Charlottesville, VA, USA). Transfectant $\mathrm{mES}$ cells line that express GFP expression driven by Oct4 promoter was performed using $4 \mu \mathrm{g}$ of linearized p-Oct4-eGFP-pgk hygro plasmid, ${ }^{40}$ cells were electroporated $(800 \mathrm{~V}, 50 \mu \mathrm{F}, 30 \mathrm{~ms}$ ) and selected by hygromycin resistense and purified by sorting. NO donor DETA-NO (Sigma, St. Louis, MO, USA), camptothecin (Calbiochem) and valproic acid (Sigma) were dissolved in PBS buffer and treatments were performed by adding the compound to the media culture. hES cells, line 181, were cultured as previously described ${ }^{39}$ on mitomycin $\mathrm{C}$-inactivated human foreskin fibroblast feeder cells (CRL-2429, ATCC) at $37^{\circ} \mathrm{C}, 5 \% \mathrm{CO}_{2}$ in DMEN/KO supplemented with $20 \%$ knockout serum replacement (Gibco-Invitrogen, CA, USA), $2 \mathrm{mM}$ L-glutamine (Gibco-Invitrogen), $0.1 \mathrm{mM}$ 2-mercaptoethanol (Gibco-Invitrogen), 1\% nonessential amino acids (Gibco-Invitrogen) and $50 \mathrm{U} / \mathrm{ml}$ and $50 \mu \mathrm{g} / \mathrm{ml}$ penicillin and streptomycin, respectively (Gibco-Invitrogen). Cells were subcultured every 10 days by mechanical splitting. For the experimental conditions described in this study, the hECS were cultured on Matrigel-coated tissue culture plates (BD Biosciences, San Jose, CA, USA) in the medium described above conditioned with human foreskin human fibroblast, in the absence or presence of $8 \mathrm{ng} / \mathrm{ml} \mathrm{bFGF}$.

Transfected D3 mES cells containing p53 stable knockdown were generated with a construct with a p53 shRNA in the pSR-GFP/Neo vector (OligoEngine, Seattle, WA, USA). This construct was provided by Dr. A Silva from the Department of Cellular and Molecular Physiopathology, CSIC, Spain. Transfections were performed with FuGENE HD Transfection Reagent (Roche Diagnostics, Mannheim, Germany) and $72 \mathrm{~h}$ later, cells were selected with $500 \mu \mathrm{g} / \mathrm{ml}$ geneticin (Sigma). Clones with stable expression of $p 53$ shRNA were picked, expanded and analyzed for p53 expression.

RNA isolation, reverse transcription (RT)-PCR and real-time PCR analysis. Total RNA was extracted using Trizol (Invitrogen) and clorophorm/ isopropanol purification procedure. cDNA synthesis was performed with $1 \mu \mathrm{g}$ of total RNA using M-MVL retrotranscriptase (Promega, Madison, WI, USA) and random primers according to the manufacturer's instructions. For real-time PCR analysis, endogenous mRNA levels were measured by real-time PCR analysis based on SYBR Green (Applied Biosystems, Foster City, CA, USA) detection with the ABI Prism 7500 machine (Applied Biosystems). Results were normalized with $\beta$-actin. Real-time PCR primers used are shown in Supplementary Table 1.

Protein extraction and western blotting. Proteins were extracted using RIPA protein extraction buffer (Sigma) supplemented with protease inhibitor (Sigma) and phosphatase inhibitor cocktails (Sigma). In brief, cells were trypsinized from culture dishes, centrifuged and washed once with cold PBS. Cell pellets were then resuspended and incubated in RIPA buffer for $20 \mathrm{~min}$ on ice and sonicated in three pulses of $10 \mathrm{~s}$ at $10 \%$ amplitude on ultrasonicater (Branson Digital Sonifier, Branson Ultrasonics Corporation, Danbury, CT, USA) to ensure high efficiency of lysis. After centrifugation, supernatants of total protein extracts were denatured in loading buffer for $10 \mathrm{~min}$ at $98^{\circ} \mathrm{C}$. Total protein $(20 \mu \mathrm{g})$ was separated using SDS-PAGE and transferred to PVDF membrane. The membrane was probed with anti-Nanog (Bethyl, Montgomery, TX, USA, BL1662), anti-Oct4 (Cell Signaling Technology, Danvers, MA, USA), anti- $\beta$-actin (Sigma), anti-p53 (Calbiochem, Ab-7), anti-phospho p53 (Ser15) (Abcam, Cambridge, UK), anti-phospho p53 (Ser315) (Thermo Scientific, Cheshire, UK), anti-phospho p53 (Ser392) (Cell Signaling Technology), anti-acetyl p53 (Lys 379) (Cell Signaling Technology), anti-histone $\mathrm{H} 3$ acetylated (Calbiochem), anti-histone $\mathrm{H} 4$ acetylated (Upstate, Lake Placid, NY, USA), anti-monomethyl histone H3 (K4) (Upstate), anti mono/di/ trimethyl histone H3 (K4) (Upstate), anti-trimethyl histone H3 (K9) (Upstate), anti-Cox IV (Abcam), anti-PCNA (Santa Cruz Biotechnologies), anti-PARP
(Cell Signaling Technology), anti-Cleaved Caspase-3 (Asp175) (Cell Signaling Technology), anti- histone H1 (Upstate) and anti- $\alpha$-tubulin (Sigma).

Nuclear, cytoplasm and mitochondrial protein extraction. Nuclear proteins were separated from cytoplasm fraction using Qproteome Nuclear Protein kit (Qiagen, Valencia, CA, USA). Mitochondrial proteins were obtained using mitochondrial isolation kit for mammalian cells (Pierce, Rockford, IL, USA).

Immunocytochemistry. Cells were fixed with methanol $\left(-20^{\circ} \mathrm{C}\right)$ for $5 \mathrm{~min}$, blocked with blocking solution for $30 \mathrm{~min}(2 \% \mathrm{BSA}, 2 \%$ Donkey serum, $2 \%$ Goat serum in PBS) and incubated overnight with anti-FoxA2 (Santa Cruz Biotechnologies, sc-6554), anti-Pdx1 (Abcam, ab47267) and anti-Sox 17 (Millipore, Billerica, MA, USA, 09-038) primary antibodies. Detection of primary antibodies was developed using appropriate secondary antibodies. Cells were counter-stained with $300 \mathrm{nM}$ DAPI. Fluorescent images were obtained using Olympus IX71 microscope (Olympus, Hamburg, Germany). Images were processed with Adobe Photoshop (Adobe Systems Incorporated, San Jose, CA, USA).

ChIP assay. In brief, cells were cross-linked with 1\% (w/v) formaldehyde for $10 \mathrm{~min}$ at $37^{\circ} \mathrm{C}$. Chromatin extracts containing DNA fragments with an average size of $500 \mathrm{bp}$ were immunoprecipitated using $4 \mu \mathrm{g}$ of antibody per sample: anti-trimethyl K9 histone H3 (Abcam; ab8898), anti-p53 (Santa Cruz Biotechnology; FL-393) and anti-p53 (Ser315) (Thermo). Anti-Rabbit lgG (Sigma) antibody was used as mock ChIP controls. ChIP assay was performed according to the manufacturer's indications using ChIP Assay kit (Upstate). Immunoprecipitated DNA was resuspended in $20 \mu \mathrm{l}$ of water and $1 \mu \mathrm{l}$ of DNA was used for PCR analysis. The following PCR primers were used:

Proximal Nanog Promoter Forward: 5'-CCCTAAGCTTTCCCTCCCTCC-3', Proximal Nanog Promoter Reverse: $5^{\prime}$-CCAAATCAGCCTATCTGAAGG-3',

Distal Nanog Promoter Forward: 5'-GGTTAGAGTGCTTTCACTCAC-3',

Distal Nanog Promoter Reverse: 5'-GCTGGCTTCAGACTTACTGC-3',

p53 element (Nanog promoter)

Forward: 5'-CAACTTACTAAGGTAGCCCGAGTCTTAA-3',

p53 element (Nanog promoter)

Reverse: 5'-CCTCCAAAAGTGCGGCTTT-3'.

Proximal Nanog Promoter amplicon, Distal Nanog Promoter amplicon and p53 element (Nanog promoter) amplicon was 242 bp, $248 \mathrm{bp}$ and $82 \mathrm{bp}$, respectively. PCR reactions were performed at $60^{\circ} \mathrm{C}$ annealing temperature for 35 cycles. PCR products were resolved in $2 \%$ agarose gels.

\section{Conflict of interest}

The authors declare no conflict of interest.

Acknowledgements. We thank $M$ Bautista and $A B$ Hitos for technical assistance and A Rojas for assistance in ChIP technique. S Mora-Castilla is fellow from Ministerio de Educación y Ciencia. This study was supported by grants from Dirección General de Investigación Científica y Técnica (SAF2007/60105), Instituto de Salud Carlos III (TERCEL RD06/0010/0025) and Junta de Andalucía (CTS576) to FJ Bedoya; from Fondo de Investigaciones Sanitarias (FIS-052106) and Dirección General de Investigación Científica y Técnica (CYT-836, SAF200508014) to B Soria; from Dirección General de Investigación Científica y Técnica (SAF2003-03307; SAF2006-06673), Junta de Andalucía (exp. 0009/06) and Instituto de Salud Carlos III (RCMN C03/08, RETIC RD06/0015/0013 and CIBERDEM) to F Martín; and from Consejería de Salud-Junta de Andalucía (PI-0095/2007) and Instituto de Salud Carlos III (CIBERDEM) to JR Tejedo.

1. Niwa H, Miyazaki J, Smith AG. Quantitative expression of Oct-3/4 defines differentiation, dedifferentiation or self-renewal of ES cells. Nat Genet 2000; 24: 372-376.

2. Mitsui K, Tokuzawa $\mathrm{Y}$, Itoh $\mathrm{H}$, Segawa $\mathrm{K}$, Murakami M, Takahashi $\mathrm{K}$ et al. The homeoprotein Nanog is required for maintenance of pluripotency in mouse epiblast and ES cells. Cell 2003; 113: 631-642.

3. Chambers I, Colby D, Robertson M, Nichols J, Lee S, Tweedie S et al. Functional expression cloning of Nanog, a pluripotency sustaining factor in embryonic stem cells. Cell 2003; 113: 643-655.

4. Boyer LA, Lee TI, Cole MF, Johnstone SE, Levine SS, Zucker JP et al. Core transcriptional regulatory circuitry in human embryonic stem cells. Cell 2005; 122: 947-956.

5. Pan G, Thomson JA. Nanog and transcriptional networks in embryonic stem cell pluripotency. Cell Res 2007; 17: 42-49. 
6. Hamazaki T, Oka M, Yamanaka S, Terada N. Aggregation of embryonic stem cells induces Nanog repression and primitive endoderm differentiation. J Cell Sci 2004; 117 (Part 23): 5681-5686.

7. Lin T, Chao C, Saito S, Mazur SJ, Murphy ME, Appella E et al. p53 induces differentiation of mouse embryonic stem cells by suppressing Nanog expression. Nat Cell Biol 2005; 7 165-171.

8. Han MK, Song EK, Guo Y, Ou X, Mantel C, Broxmeyer HE. SIRT1 regulates apoptosis and Nanog expression in mouse embryonic stem cells by controlling p53 subcellular localization. Cell Stem Cell 2008; 2: 241-251.

9. Gu W, Roeder RG. Activation of $p 53$ sequence-specific DNA binding by acetylation of the p53 C-terminal domain. Cell 1997; 90: 595-606.

10. Lee JH, Hart SR, Skalnik DG. Histone deacetylase activity is required for embryonic stem cell differentiation. Genesis 2004; 38: 32-38.

11. Loh YH, Zhang W, Chen X, George J, Ng HH. Jmjd1a and Jmjd2c histone H3 Lys demethylases regulate self-renewal in embryonic stem cells. Genes Dev2007; 21: 2545-2557.

12. Kuo RC, Baxter GT, Thompson SH, Stricker SA, Patton C, Bonaventura J et al. NO is necessary and sufficient for egg activation at fertilization. Nature 2000; 406: 633-636.

13. Sengoku K, Takuma N, Horikawa M, Tsuchiya K, Komori H, Sharifa D et al. Requirement of nitric oxide for murine oocyte maturation, embryo development, and trophoblast outgrowth in vitro. Mol Reprod Dev 2001; 58: 262-268.

14. Gouge RC, Marshburn P, Gordon BE, Nunley W, Huet-Hudson YM. Nitric oxide as a regulator of embryonic development. Biol Reprod 1998; 58: 875-879.

15. Kanno S, Kim PK, Sallam K, Lei J, Billiar TR, Shears II LL. Nitric oxide facilitates cardiomyogenesis in mouse embryonic stem cells. Proc Natl Acad Sci USA 2004; 101: 12277-12281.

16. Paquin J, Danalache BA, Jankowski M, McCann SM, Gutkowska J. Oxytocin induces differentiation of P19 embryonic stem cells to cardiomyocytes. Proc Natl Acad Sci USA 2002; 99: 9550-9555.

17. Danalache BA, Paquin J, Donghao W, Grygorczyk R, Moore JC, Mummery CL et al. Nitric oxide signaling in oxytocin-mediated cardiomyogenesis. Stem cells (Dayton, $\mathrm{OH}$ ) 2007; 25 679-688.

18. Gassanov N, Jankowski M, Danalache B, Wang D, Grygorczyk R, Hoppe UC et al. Arginine vasopressin-mediated cardiac differentiation: insights into the role of its receptors and nitric oxide signaling. J Biol Chem 2007; 282: 11255-11265.

19. Bloch W, Fleischmann BK, Lorke DE, Andressen C, Hops B, Hescheler J et al. Nitric oxide synthase expression and role during cardiomyogenesis. Cardiovasc Res 1999; 43 : 675-684.

20. Mujoo K, Sharin VG, Bryan NS, Krumenacker JS, Sloan C, Parveen S et al. Role of nitric oxide signaling components in differentiation of embryonic stem cells into myocardial cells. Proc Natl Acad Sci USA 2008; 105: 18924-18929.

21. Wang X, Michael D, de Murcia G, Oren M. p53 Activation by nitric oxide involves downregulation of Mdm2. J Biol Chem 2002; 277: 15697-15702.

22. Yung HW, Bal-Price AK, Brown GC, Tolkovsky AM. Nitric oxide-induced cell death of cerebrocortical murine astrocytes is mediated through p53- and Bax-dependent pathways J Neurochem 2004; 89: 812-821.

23. McLaughlin LM, Demple B. Nitric oxide-induced apoptosis in lymphoblastoid and fibroblast cells dependent on the phosphorylation and activation of p53. Cancer Res 2005 65: 6097-6104.
24. Qin $\mathrm{H}, \mathrm{Yu} \mathrm{T}$, Qing T, Liu Y, Zhao Y, Cai J et al. Regulation of apoptosis and differentiation by $\mathrm{p} 53$ in human embryonic stem cells. J Biol Chem 2007; 282: 5842-5852.

25. Hamazaki T, Kehoe SM, Nakano T, Terada N. The Grb2/Mek pathway represses Nanog in murine embryonic stem cells. Mol Cell Biol 2006; 26: 7539-7549.

26. Loh YH, Wu Q, Chew JL, Vega VB, Zhang W, Chen X et al. The Oct4 and Nanog transcription network regulates pluripotency in mouse embryonic stem cells. Nat Genet 2006; 38: 431-440.

27. Guo Y, Costa R, Ramsey H, Starnes T, Vance G, Robertson K et al. The embryonic stem cell transcription factors Oct-4 and FoxD3 interact to regulate endodermal-specific promoter expression. Proc Natl Acad Sci USA 2002; 99: 3663-3667.

28. Maimets T, Neganova I, Armstrong L, Lako M. Activation of p53 by nutlin leads to rapid differentiation of human embryonic stem cells. Oncogene 2008; 27: 5277-5287.

29. Kroon E, Martinson LA, Kadoya K, Bang AG, Kelly OG, Eliazer S et al. Pancreatic endoderm derived from human embryonic stem cells generates glucose-responsive insulin-secreting cells in vivo. Nat Biotechnol 2008; 26: 443-452.

30. D'Amour KA, Agulnick AD, Eliazer S, Kelly OG, Kroon E, Baetge EE. Efficient differentiation of human embryonic stem cells to definitive endoderm. Nat Biotechnol 2005; 23: $1534-1541$.

31. D'Amour KA, Bang AG, Eliazer S, Kelly OG, Agulnick AD, Smart NG et al. Production of pancreatic hormone-expressing endocrine cells from human embryonic stem cells. Nat Biotechnol 2006; 24: 1392-1401.

32. Lavin MF, Gueven N. The complexity of p53 stabilization and activation. Cell Death Differ 2006; 13: 941-950.

33. Blaydes JP, Luciani MG, Pospisilova S, Ball HM, Vojtesek B, Hupp TR. Stoichiometric phosphorylation of human p53 at Ser315 stimulates p53-dependent transcription. J Biol Chem 2001; 276: 4699-4708

34. Nemajerova A, Erster S, Moll UM. The post-translational phosphorylation and acetylation modification profile is not the determining factor in targeting endogenous stress-induced p53 to mitochondria. Cell Death Differ 2005; 12: 197-200.

35. Francis NJ, Kingston RE. Mechanisms of transcriptional memory. Nat Rev 2001; 2 409-421.

36. Haumaitre $\mathrm{C}$, Lenoir $\mathrm{O}$, Scharfmann $\mathrm{R}$. Histone deacetylase inhibitors modify pancreatic cell fate determination and amplify endocrine progenitors. Mol Cell Biol 2008; 28: 6373-6383.

37. Vaca P, Berna G, Araujo R, Carneiro EM, Bedoya FJ, Soria B et al. Nicotinamide induces differentiation of embryonic stem cells into insulin-secreting cells. Exp Cell Res 2008; 314: 969-974.

38. Goicoa S, Alvarez S, Ricordi C, Inverardi L, Dominguez-Bendala J. Sodium butyrate activates genes of early pancreatic development in embryonic stem cells. Cloning Stem Cells 2006; 8: 140-149.

39. Hovatta O, Mikkola M, Gertow K, Stromberg AM, Inzunza J, Hreinsson J et al. A culture system using human foreskin fibroblasts as feeder cells allows production of human embryonic stem cells. Hum Reprod (Oxford, England) 2003; 18: 1404-1409.

40. Ensenat-Waser R, Santana A, Vicente-Salar N, Cigudosa JC, Roche E, Soria B et al. Isolation and characterization of residual undifferentiated mouse embryonic stem cells from embryoid body cultures by fluorescence tracking. In Vitro Cell Dev Biol Anim 2006; 42 : $115-123$.

Supplementary Information accompanies the paper on Cell Death and Differentiation website (http://www.nature.com/cdd) 